\title{
SCHWARZ'S LEMMA AND THE KOBAYASHI AND CARATHÉODORY METRICS ON COMPLEX BANACH MANIFOLDS
}

\author{
CLIFFORD J. EARLE, LAWRENCE A. HARRIS, JOHN H. HUBBARD, \\ AND SUDEB MITRA
}

\begin{abstract}
We discuss the Carathéodory and Kobayashi pseudometrics and their infinitesimal forms on complex Banach manifolds. Our discussiion includes a very elementary treatment of the Kobayashi pseudometric as an integral of its infinitesimal form. We also prove new distortion theorems for the Carathéodory pseudometric under holomorphic maps from the open unit disk to a complex Banach manifold.
\end{abstract}

\section{INTRODUCTION}

The geometry of the hyperbolic plane has played an important role in complex function theory ever since the time of Poincaré, who brilliantly exploited the fact that the open unit disk $\mathbb{D}$ in the complex plane $\mathbb{C}$ carries a hyperbolic metric (commonly called the Poincaré metric) that is preserved by all holomorphic automorphisms of $\mathbb{D}$. This metric therefore descends to a hyperbolic metric on any Riemann surface whose universal covering surface is holomorphically isomorphic to $\mathbb{D}$, so (by the uniformization theorem) almost all Riemann surfaces carry natural complete hyperbolic metrics.

A further link between function theory and the hyperbolic geometry of the unit disk is provided by the Schwarz-Pick lemma. It states that a holomorphic map of $\mathbb{D}$ into itself does not increase the hyperbolic lengths of tangent vectors or the Poincaré distances between points. This result also descends to Riemann surfaces whose universal covering surface is holomorphically isomorphic to $\mathbb{D}$. If $X$ and $Y$ are such surfaces with their natural hyperbolic metrics then a holomorphic map from $X$ to $Y$ does not increase the hyperbolic lengths of tangent vectors or the hyperbolic distances between points.

No higher dimensional complex manifold has the privileged position that $\mathbb{D}$ occupies in the one-dimensional case, so the study of natural metrics on such manifolds continues to be an active area of research. In particular, systems of pseudometrics on complex spaces that satisfy the Schwarz-Pick lemma have been much studied. See for example the papers [Har79], [Kob67], [PSh89], [Roy88], and [Ven89] and the books [Din89], [FV80], [JP93], and [Kob98]. In [Roy88] Royden calls such systems hyperbolic metrics, which partly explains the presence of this paper in this volume.

The Carathéodory and Kobayashi pseudometrics are the most widely studied of these systems, and they are our focus of attention. In section 3 we combine results of Harris [Har79] with an idea of Royden [Roy88] to give a very elementary proof that the Kobayashi pseudometric can be defined by appropriately integrating an associated infinitesimal metric. Sharper results that apply to a more general class 
of pseudometrics can be found in Venturini's incisive paper [Ven89], which uses less elementary methods and places the results of [Har79] in a broader setting.

In section 4 we shall define both the Carathéodory pseudometric and an associated inner pseudometric, and in section 5 we shall obtain sharp new forms of the Schwarz-Pick lemma for holomorphic maps from $\mathbb{D}$ to $X$ equipped with either of these pseudometrics. These results describe in quantitative terms how close a holomorphic map from $\mathbb{D}$ to $X$ comes to being a complex Carathéodory geodesic. They are inspired by and derived from results in the beautiful paper [BMi] of Beardon and Minda about holomorphic maps of $\mathbb{D}$ into itself. We thank David Minda for sending us a preprint of [BMi] and for an additional private communication that led us to formulate and prove Theorem 5.5.

\section{SCHWARZ-PiCK SYSTEMS}

General systems of pseudometrics with the Schwarz-Pick property were first studied systematically in [Har79] by Harris, who coined the term Schwarz-Pick system for them. These systems can be studied on various classes of complex spaces. We choose to use the class of complex manifolds modelled on complex Banach spaces of positive, possibly infinite, dimension. All our manifolds are assumed to be connected Hausdorff spaces.

If $X$ and $Y$ are complex Banach manifolds, we shall denote the set of all holomorphic maps of $X$ into $Y$ by $\mathcal{O}(X, Y)$.

Definition 2.1. (see Harris [Har79]) A Schwarz-Pick system is a functor, denoted by $X \mapsto d_{X}$, that assigns to each complex Banach manifold $X$ a pseudometric $d_{X}$ so that the following conditions hold:

(a) The pseudometric assigned to $\mathbb{D}$ is the Poincaré metric

$$
d_{\mathbb{D}}\left(z_{1}, z_{2}\right)=\tanh ^{-1}\left|\frac{z_{1}-z_{2}}{1-z_{1} \overline{z_{2}}}\right| \text { if } z_{1} \in \mathbb{D} \text { and } z_{2} \in \mathbb{D} .
$$

(b) If $X$ and $Y$ are complex Banach manifolds then

$$
d_{Y}\left(f\left(x_{1}\right), f\left(x_{2}\right)\right) \leq d_{X}\left(x_{1}, x_{2}\right) \text { if } x_{1} \in X, x_{2} \in X \text { and } f \in \mathcal{O}(X, Y) .
$$

Remark 2.2. Because of conditions (a) and (b) the sets $\mathcal{O}(\mathbb{D}, X)$ and $\mathcal{O}(X, \mathbb{D})$ provide upper and lower bounds for $d_{X}$. These upper and lower bounds lead to the definitions of the Kobayashi and Carathéodory pseudometrics, which we shall study in the remainder of this paper.

\section{The Kobayashi PSEUdometric And its InfInitesimal FORM}

3.1. The classical definition. In this paper $d_{\mathbb{D}}$ will always be the Poincaré metric (2.1) on the unit disk $\mathbb{D}$.

Definition 3.1. A Schwarz-Pick pseudometric on the complex Banach manifold $X$ is a pseudometric $d$ such that

$$
d(f(z), f(w)) \leq d_{\mathbb{D}}(z, w) \quad \text { for all } z \text { and } w \text { in } \mathbb{D} \text { and } f \text { in } \mathcal{O}(\mathbb{D}, X) .
$$

If $X \mapsto d_{X}$ is a Schwarz-Pick system, then $d_{X}$ is obviously a Schwarz-Pick pseudometric on $X$ for every complex Banach manifold $X$.

Definition 3.2. The Kobayashi pseudometric $K_{X}$ is the largest Schwarz-Pick pseudometric on the complex Banach manifold $X$. 
As Kobayashi observed (see for example [Kob67] or [Kob98]), $K_{X}$ is easily described in terms of the function $\delta_{X}: X \times X \rightarrow[0, \infty]$ defined by

$$
\delta_{X}\left(x, x^{\prime}\right)=\inf \left\{d_{\mathbb{D}}(0, z): x=f(0) \text { and } x^{\prime}=f(z) \text { for some } f \in \mathcal{O}(\mathbb{D}, X)\right\}
$$

for all $x$ and $x^{\prime}$ in $X$. (As usual the infimum of the empty set is $\infty$.)

In fact (3.1), the definition of $\delta_{X}$, and the triangle inequality imply that any Schwarz-Pick pseudometric $d$ on $X$ satisfies

$$
d\left(x, x^{\prime}\right) \leq \inf \left\{\sum_{j=1}^{n} \delta_{X}\left(x_{j-1}, x_{j}\right)\right\} \quad \text { for all } x \text { and } x^{\prime} \text { in } X,
$$

where the infimum is taken over all positive integers $n$ and all $(n+1)$-tuples of points $x_{0}, \ldots, x_{n}$ in $X$ such that $x_{0}=x$ and $x_{n}=x^{\prime}$.

The infimum on the right side of the inequality (3.2) defines a function on $X \times X$ that is obviously a Schwarz-Pick pseudometric on $X$, so (3.2) implies that

$$
K_{X}\left(x, x^{\prime}\right)=\inf \left\{\sum_{j=1}^{n} \delta_{X}\left(x_{j-1}, x_{j}\right)\right\} \quad \text { for all } x \text { and } x^{\prime} \text { in } X .
$$

(The infimum is of course taken over the same set as in (3.2) above.)

Equation (3.3) is Kobayashi's definition of the pseudometric $K_{X}$. It follows readily from (3.3) and the Schwarz-Pick lemma that the functor assigning the Kobayashi pseudometric $K_{X}$ to each complex Banach manifold $X$ is a SchwarzPick system. A slightly stronger property of this functor will follow from the arc length description of $K_{X}$ that we shall explain in the remainder of section 2.

3.2. The infinitesimal Kobayashi pseudometric and its integrated form. Every complex Banach manifold $X$ has an infinitesimal Kobayashi pseudometric $k_{X}$, first introduced (in the finite dimensional case) by Kobayashi in [Kob67]. Since $k_{X}$ is a function on the tangent bundle $T(X)$ of $X$, we shall briefly review some properties of tangent bundles.

For the moment let $X$ be a $C^{1}$ manifold modelled on a real Banach space $V$. For each $x$ in $X$ the tangent space to $X$ at $x$ will be denoted by $T_{x}(X)$. The tangent bundle $T(X)$ of $X$ consists of the ordered pairs $(x, v)$ such that $x \in X$ and $v \in T_{x}(X)($ see $[\operatorname{Lan} 62])$.

If $X$ is an open set in $V$ with the $C^{1}$ structure induced by the inclusion map, then each $T_{x}(X), x$ in $X$, is naturally identified with $V$, and $T(X)=X \times V$.

If $X$ and $Y$ are $C^{1}$ manifolds and $x$ is a point of $X$, every $C^{1}$ map $f: X \rightarrow Y$ induces a linear map $f_{*}(x)$ from $T_{x}(X)$ to $T_{f(x)}(Y)$ (see [Lan62]). If $X$ and $Y$ are subregions of Banach spaces $V$ and $W$ and the tangent spaces $T_{x}(X)$ and $T_{f(x)}(Y)$ are identified with $V$ and $W$ in the natural way, then $f_{*}(x)$ is the usual Fréchet derivative of $f$ at $x$.

The tangent bundle $T(X)$ has a natural $C^{0}$ manifold structure modelled on $V \times V$. A convenient atlas for $T(X)$ consists of the charts $T(\varphi)$ defined by the formula

$$
T(\varphi)(x, v)=\left(\varphi(x), \varphi_{*}(x) v\right), \quad(x, v) \in T(U),
$$

where $U$ is an open set in $X, T(U)$ is the open subset $\{(x, v) \in T(X): x \in U\}$ of $T(X)$, and $\varphi$ is a chart on $X$ with domain $U$. The image of $T(U)$ under $T(\varphi)$ is the open set $\varphi(U) \times V$ in $V \times V$. 
If $X$ is a complex Banach manifold modelled on a complex Banach space $V$, then each tangent space $T_{x}(X)$ has a unique complex Banach space structure such that the map $\varphi_{*}(x)$ from $T_{x}(X)$ to $V$ is a $\mathbb{C}$-linear isomorphism whenever $\varphi$ is a (holomorphic) chart defined in some neighborhood of $x$. Furthermore, $T(X)$ has a unique complex Banach manifold structure such that the map $T(\varphi)$ from $T(U)$ to $\varphi(U) \times V$ is biholomorphic for every (holomorphic) chart $\varphi$ on $X$ with domain $U$ (see $[$ Dou66]).

Now we are ready for Kobayashi's definition of $k_{X}$.

Definition 3.3. The infinitesimal Kobayashi pseudometric on the complex Banach manifold $X$ is the function $k_{X}$ on $T(X)$ defined by the formula

$$
k_{X}(x, v)=\inf \left\{|z|: x=f(0) \text { and } v=f_{*}(0) z \text { for some } f \in \mathcal{O}(\mathbb{D}, X)\right\} .
$$

Obviously $k_{X}(x, v) \geq 0$ and $k_{X}(x, c v)=|c| k_{X}(x, v)$ for all complex numbers $c$. The following Schwarz-Pick property is also an immediate consequence of the definition (see [Roy88] or Theorem 1.2.6 in [NO90]).

Proposition 3.4. If $X$ and $Y$ are complex Banach manifolds and $f \in \mathcal{O}(X, Y)$, then

$$
k_{Y}\left(f(x), f_{*}(x) v\right) \leq k_{X}(x, v) \quad \text { for all }(x, v) \in T(X) .
$$

In particular, if $f$ is biholomorphic then $k_{Y}\left(f(x), f_{*}(x) v\right)=k_{X}(x, v)$.

Corollary 3.5. $k_{\mathbb{D}}(w, z)=\frac{|z|}{1-|w|^{2}}$ for all $(w, z)$ in $\mathbb{D} \times \mathbb{C}$.

Proof. Definition 3.3 and Schwarz's lemma imply that $k_{\mathbb{D}}(0, z)=|z|$ for all complex numbers $z$. To prove the formula for $k_{\mathbb{D}}(w, z)$, apply Proposition 3.4 with $X=Y=$ $\mathbb{D}$ and $f(\zeta)=(\zeta-w) /(1-\zeta \bar{w}), \zeta$ in $\mathbb{D}$.

We shall use the function $k_{X}$ to measure the lengths of piecewise $C^{1}$ curves in $X$. As usual, if the curve $\gamma:[a, b] \rightarrow X$ is differentiable at $t$ in $[a, b]$ the symbol $\gamma^{\prime}(t)$ denotes the tangent vector $\gamma_{*}(t) 1$ to $X$ at $\gamma(t)$. If $\gamma$ is piecewise $C^{1}$, it is natural to define the Kobayashi length of $\gamma$ by integrating the function $k_{X}\left(\gamma(t), \gamma^{\prime}(t)\right)$ over the parameter interval of $\gamma$. That function is upper semicontinuous when $X$ is either a domain in a complex Banach space (see [Har79] or [Din89]) or a finite dimensional complex manifold (see [Roy71] and [Roy74] or [NO90]), but the case of infinite dimensional complex manifolds is harder to deal with. In [Roy88] Royden evades that difficulty by using the upper Riemann integral. Venturini [Ven89] gets more refined results by using upper and lower Lebesgue integrals. We shall follow Royden's example, as it allows the very elementary arguments that we shall now present.

The required upper Riemann integrals exist because the function $k_{X}$ is locally bounded on $T(X)$. To prove this we use special charts on $X$. By definition, a standard chart at $x$ in $X$ is a biholomorphic map $\varphi$ of an open neighborhood of $x$ onto the open unit ball of $V$ with $\varphi(x)=0$.

Lemma 3.6. If $\varphi$ is a standard chart at the point $x$ in $X$, then

$$
k_{X}(y, v) \leq 2\left\|\varphi_{*}(y) v\right\|
$$

for all $(y, v)$ in $T(X)$ such that $y$ is in the domain of $\varphi$ and $\|\varphi(y)\| \leq 1 / 2$. 
Proof. If $v=0$ the inequality is trivial. If $v \neq 0$ we derive it from (3.4) by setting

$$
f(z)=\varphi^{-1}\left(\varphi(y)+z \frac{\varphi_{*}(y) v}{2\left\|\varphi_{*}(y) v\right\|}\right), \quad z \in \mathbb{D},
$$

so that $f \in \mathcal{O}(\mathbb{D}, X), f(0)=y$, and $f_{*}(0) c=\frac{c}{2\left\|\varphi_{*}(y) v\right\|} v$ for all $c$ in $\mathbb{C}$.

Corollary 3.7. The function $k_{X}$ is locally bounded in $T(X)$.

Proof. Let $U$ be the domain of the standard chart $\varphi$ in Lemma 3.6. Since holomorphic maps are $C^{1}$, the function $(y, v) \mapsto 2\left\|\varphi_{*}(y) v\right\|$ is locally bounded in the open set $T(U)=\{(y, v) \in T(X): y \in U\}$.

Following Royden [Roy88], we can now define the arc length $L_{X}(\gamma)$ of a piecewise $C^{1}$ curve $\gamma:[a, b] \rightarrow X$ in $X$ to be the upper Riemann integral

$$
L_{X}(\gamma)=\bar{\int}_{a}^{b} k_{X}\left(\gamma(t), \gamma^{\prime}(t)\right) d t
$$

and the distance $\rho_{X}(x, y)$ to be the infimum of the lengths of all piecewise $C^{1}$ curves joining $x$ to $y$ in $X$. The resulting pseudometric $\rho_{X}$ on $X$ is the integrated form of the Kobayashi infinitesimal pseudometric $k_{X}$. By Proposition 3.4,

$$
\rho_{Y}\left(f\left(x_{1}\right), f\left(x_{2}\right)\right) \leq \rho_{X}\left(x_{1}, x_{2}\right) \quad \text { for all } x_{1} \text { and } x_{2} \text { in } X
$$

whenever $f \in \mathcal{O}(X, Y)$. In fact even more is true. If $f \in \mathcal{O}(X, Y)$ and $\gamma$ is a piecewise $C^{1}$ curve in $X$, then $L_{Y}(f \circ \gamma) \leq L_{X}(\gamma)$.

Remark 3.8. The upper Riemann integral of an upper semicontinuous function equals its Lebesgue integral, so we can use a Lebesgue integral in (3.5) if $X$ is finite dimensional or a region in a complex Banach space, but that is an unnecessary luxury.

Remark 3.9. By Corollary $3.5, \rho_{\mathbb{D}}$ is the Poincaré metric $d_{\mathbb{D}}$ on $\mathbb{D}$. Therefore, by (3.6), the functor that assigns $\rho_{X}$ to each complex Banach manifold $X$ is a Schwarz-Pick system. In particular $\rho_{X}$ is a Schwarz-Pick pseudometric on $X$, so $\rho_{X}(x, y) \leq K_{X}(x, y)$ for all $x$ and $y$ in $X$. In the next subsection we shall use methods of Harris [Har79] to prove that the pseudometrics $\rho_{X}$ and $K_{X}$ are in fact equal.

3.3. Upper bounds for Schwarz-Pick pseudometrics. We begin with a simple estimate.

Lemma 3.10. Let d be a Schwarz-Pick pseudometric on $X$ and let $\varphi$ be a standard chart at the point $x_{0}$ in $X$. If $0<r<1 / 3$ there is a constant $C(r)$ such that

$$
d(x, y) \leq C(r)\|\varphi(x)-\varphi(y)\|
$$

for all $x$ and $y$ in the domain of $\varphi$ such that $\|\varphi(x)\| \leq r$ and $\|\varphi(y)\| \leq r$.

Proof. There is nothing to prove if $x=y$. If $x, y$ and $r$ satisfy the stated conditions and $x \neq y$, define $f$ in $\mathcal{O}(\mathbb{D}, X)$ by

$$
f(z)=\varphi^{-1}\left(\varphi(x)+(1-r) z \frac{\varphi(y)-\varphi(x)}{\|\varphi(y)-\varphi(x)\|}\right), \quad z \in \mathbb{D} .
$$


Our hypothesis implies that $z_{0}=\|\varphi(y)-\varphi(x)\| /(1-r)$ belongs to $\mathbb{D}$. Since $f\left(z_{0}\right)=y$ and $f(0)=x$, inequality $(3.1)$ gives

$$
d(x, y) \leq d_{\mathbb{D}}(0,\|\varphi(y)-\varphi(x)\| /(1-r)) .
$$

Since $d_{\mathbb{D}}(0, s) / s$ is an increasing function of $s$ in the interval $(0,1)$ the required inequality holds with $C(r)=d_{\mathbb{D}}(0,2 r /(1-r)) / 2 r$.

The crucial step is the following result from [Har79] (see pp. 368 and 371).

Lemma 3.11 (Harris). Let $\gamma:[a, b] \rightarrow X$ be a $C^{1}$ curve in $X$. If $d$ is a SchwarzPick metric on $X$ then

$$
\limsup _{t \rightarrow s} \frac{d(\gamma(t), \gamma(s))}{|t-s|} \leq k_{X}\left(\gamma(s), \gamma^{\prime}(s)\right) \quad \text { for all } s \in[a, b]
$$

Proof. Choose $s$ in $[a, b]$ and a standard chart $\varphi$ at $\gamma(s)$. Let $c$ be a complex number such that there is $f$ in $\mathcal{O}(\mathbb{D}, X)$ with $f(0)=\gamma(s)$ and $f_{*}(0) c=\gamma^{\prime}(s)$.

Since the curves $t \mapsto \varphi(\gamma(t))$ and $t \mapsto \varphi(f(c(t-s)))$ are tangent at $t=s$,

$$
\varphi(\gamma(t))-\varphi(f(c(t-s)))=o(t-s)
$$

as $t$ approaches $s$ in $[a, b]$. Therefore, by Lemma 3.10 and inequality (3.1),

$$
d(\gamma(t), \gamma(s)) \leq d(f(c(t-s)), f(0))+o(t-s) \leq d_{\mathbb{D}}(c(t-s), 0)+o(t-s)
$$

and

$$
\limsup _{t \rightarrow s} \frac{d(\gamma(t), \gamma(s))}{|t-s|} \leq \limsup _{t \rightarrow s} \frac{d_{\mathbb{D}}(c(t-s), 0)}{|t-s|}=|c| .
$$

Since $k_{X}\left(\gamma(s), \gamma^{\prime}(s)\right)$ is the infimum of all such complex numbers $|c|$, the lemma is proved.

We also need a simple fact from integration theory. (See pp. 369 and 370 of [Har79] for a proof that uses only the Riemann integrability of the positive function h.)

Lemma 3.12. Let $h$ be a positive continuous function on the closed interval $[a, b]$ and let $\rho$ be a pseudometric on $[a, b]$. If

$$
\limsup _{t \rightarrow s} \frac{\rho(s, t)}{|s-t|} \leq h(s) \quad \text { for all } s \in[a, b],
$$

then $\rho(a, b) \leq \int_{a}^{b} h(t) d t$.

Proof. Set $J=\rho(a, b) / \int_{a}^{b} h$. If $a \leq x<c<y \leq b$ and $\rho(x, y) \geq J \int_{x}^{y} h$ then, by the triangle inequality, either $\rho(x, c) \geq J \int_{x}^{c} h$ or $\rho(c, y) \geq J \int_{c}^{y} h$.

We can therefore inductively define sequences $\left\{x_{n}\right\}$ and $\left\{y_{n}\right\}$ in $[a, b]$ such that $x_{1}=a, y_{1}=b, x_{n} \leq x_{n+1}<y_{n+1} \leq y_{n},\left(y_{n+1}-x_{n+1}\right)=\frac{1}{2}\left(y_{n}-x_{n}\right)$, and

$$
\rho\left(x_{n}, y_{n}\right) \geq J \int_{x_{n}}^{y_{n}} h(t) d t
$$

for every $n$. Let $s=\lim _{n \rightarrow \infty} x_{n}=\lim _{n \rightarrow \infty} y_{n}$. For each $n$ we can choose $t_{n}$ equal to one of the points $x_{n}$ or $y_{n}$ so that $t_{n} \neq s$ and

$$
\frac{\rho\left(s, t_{n}\right)}{\left|s-t_{n}\right|} \geq J\left|\frac{\int_{s}^{t_{n}} h(t) d t}{s-t_{n}}\right| .
$$


As $n \rightarrow \infty$ the right side of this inequality converges to $J h(s)$. By hypothesis $h(s)>0$ and the lim sup of the left side is at most $h(s)$, so $J \leq 1$.

The following theorem, which follows readily from Lemmas 3.11 and 3.12 , is a special case of Theorem 3.1 of Venturini [Ven89]. Venturini's treatment of arc length uses both a more refined theory of integration and a wider class of admissible curves.

Theorem 3.13. If $d$ is a Schwarz-Pick metric on $X$, then $d(x, y) \leq \rho_{X}(x, y)$ for all $x$ and $y$ in $X$.

Proof. We follow the proof of Proposition 14 in [Har79]. It suffices to prove that $d(\gamma(a), \gamma(b)) \leq L_{X}(\gamma)$ for all $C^{1}$ curves $\gamma:[a, b] \rightarrow X$. By definition

$$
L_{X}(\gamma)=\bar{\int}_{a}^{b} f(t) d t
$$

with $f(t)=k_{X}\left(\gamma(t), \gamma^{\prime}(t)\right)$ for $t$ in $[a, b]$.

Let $\epsilon>0$ be given. By the definition of upper Riemann sums and integrals there is a continuous function $h$ on $[a, b]$ such that $h(t)>f(t)(\geq 0)$ for all $t$ in $[a, b]$ and

$$
\int_{a}^{b} h(t) d t<L_{X}(\gamma)+\epsilon
$$

Consider the pseudometric $\rho(s, t)=d(\gamma(s), \gamma(t))$ on $[a, b]$. By Lemma 3.11, $\rho$ and $h$ satisfy (3.7), so Lemma 3.12 and the inequality (3.8) give

$$
d(\gamma(a), \gamma(b))=\rho(a, b) \leq \int_{a}^{b} h(t) d t<L_{X}(\gamma)+\epsilon .
$$

Since $\epsilon$ is arbitrary the proof is complete.

Corollary 3.14. The pseudometric $\rho_{X}$ is the Kobayashi pseudometric on $X$.

This is obvious since Theorem 3.13 identifies $\rho_{X}$ as the largest Schwarz-Pick pseudometric on $X$. We conclude that $K_{X}$ is the integrated form of the infinitesimal pseudometric $k_{X}$.

\section{The Carathéodory PSeudometric and its infinitesimal Form}

4.1. The definitions. Let $x$ and $y$ be points of the complex Banach manifold $X$ and let $v$ be a tangent vector to $X$ at $x$. Since the Kobayashi pseudometrics form a Schwarz-Pick system, Definition 2.1 implies that

$$
d_{\mathbb{D}}(f(x), f(y)) \leq K_{X}(x, y) \quad \text { for all } f \text { in } \mathcal{O}(X, \mathbb{D}) .
$$

Similarly, Proposition 3.4 and Corollary 3.5 give

$$
\left|f_{*}(x) v\right| \leq \frac{\left|f_{*}(x) v\right|}{1-|f(x)|^{2}} \leq k_{X}(x, v) \quad \text { for all } f \text { in } \mathcal{O}(X, \mathbb{D}) .
$$

Therefore the numbers

$$
C_{X}(x, y)=\sup \left\{d_{\mathbb{D}}(f(x), f(y)): f \in \mathcal{O}(X, \mathbb{D})\right\}
$$

and

$$
c_{X}(x, v)=\sup \left\{\left|f_{*}(x) v\right|: f \in \mathcal{O}(X, \mathbb{D})\right\}
$$

are finite and are bounded by $K_{X}(x, y)$ and $k_{X}(x, v)$ respectively. 
By definition, $C_{X}(x, y)$ is the Carathéodory pseudo-distance between $x$ and $y$, and $c_{X}(x, v)$ is the Carathéodory length of $v$. The functions $C_{X}$ and $c_{X}$ are called the Carathéodory pseudometric and the infinitesimal Carathéodory pseudometric respectively.

When $X$ is the unit disk $\mathbb{D}$, we can take $f$ in $\mathcal{O}(\mathbb{D}, \mathbb{D})$ to be the identity map and obtain

$$
d_{\mathbb{D}}(z, w) \leq C_{\mathbb{D}}(z, w) \leq K_{\mathbb{D}}(z, w)=d_{\mathbb{D}}(z, w) \quad \text { for all } z \text { and } w \text { in } \mathbb{D}
$$

from (4.3) and (4.1).

Similarly, given any $w$ in $\mathbb{D}$, we use the map $\zeta \mapsto(\zeta-w) /(1-\zeta \bar{w})$ in $\mathcal{O}(\mathbb{D}, \mathbb{D})$ to obtain

$$
\frac{|z|}{1-|w|^{2}} \leq c_{\Delta}(w, z) \leq k_{\mathbb{D}}(w, z)=\frac{|z|}{1-|w|^{2}} \quad \text { for all } z \text { in } \mathbb{C}
$$

from (4.4)), (4.2), and Corollary 3.5. These observations verify the well-known fact that the functions $C_{\mathbb{D}}$ and $c_{\mathbb{D}}$ are respectively the Poincaré metric and the infinitesimal Poincaré metric on $\mathbb{D}$.

It is evident from the definition (4.3) that the functor assigning the Carathéodory pseudometric $C_{X}$ to each complex Banach manifold $X$ is a Schwarz-Pick system. This Carathéodory functor is the first Schwarz-Pick system, having been introduced by Carathéodory in [Car26] for domains in $\mathbb{C}^{2}$.

4.2. The derivative of $C_{X}$. It is well known (see for example [Din89], [Har79], [JP93], or [Ven89]) that the function $c_{X}$ is continuous on $T(X)$ and is the derivative of $C_{X}$ in the sense that

$$
\lim _{t \rightarrow 0} \frac{C_{X}(\gamma(0), \gamma(t))}{|t|}=c_{X}\left(\gamma(0), \gamma^{\prime}(0)\right)
$$

whenever $\gamma:(-\epsilon, \epsilon) \rightarrow X$ is a $C^{1}$ curve in $X$. Since the cited references provide detailed proofs of (4.5) only when $X$ is an open subset of $V$, we shall prove (4.5) here by extending the treatment of $C_{X}$ and $c_{X}$ in [EH70] to the manifold case. Our proof will show that (4.5) also holds when $t$ is a complex variable and $\gamma(t)$ is a holomorphic map of a neighborhood of 0 into $X$ (see Corollary 4.5 below).

We need two well-known lemmas.

Lemma 4.1. Let $H^{\infty}(X)$ be the Banach space of bounded holomorphic functions on $X$, and let $H^{\infty}(X)^{*}$ be its dual space. The map $\phi: X \rightarrow H^{\infty}(X)^{*}$ defined by

$$
\phi(x)(f)=f(x), \quad x \in X \text { and } f \in H^{\infty}(X),
$$

is holomorphic.

Proof. Fix $x_{0}$ in $X$ and a neighborhood $U$ of $x_{0}$ that is biholomorphically equivalent to a bounded open set in $V$. It follows readily from Cauchy's estimates that the formula $\phi_{U}(x)(f)=f(x), x$ in $U$ and $f$ in $H^{\infty}(U)$, defines a holomorphic embedding of $U$ in $H^{\infty}(U)^{*}$. (See [EH70] for details.) The restriction of $\phi$ to $U$ is the composition of $\phi_{U}$ with the bounded linear map from $H^{\infty}(U)^{*}$ to $H^{\infty}(X)^{*}$ that takes $\ell$ in $H^{\infty}(U)^{*}$ to the linear functional $f \mapsto \ell(f \mid U)$ on $H^{\infty}(X)$, so $\phi$ is holomorphic in $U$.

Corollary 4.2. If $x$ is a point of $X$ and $v$ is a tangent vector to $X$ at $x$, then the Carathéodory length $c_{X}(x, v)$ of $v$ equals the norm $\left\|\phi_{*}(x) v\right\|$ of the linear functional $\phi_{*}(x) v$ on $H^{\infty}(X)$. In particular, $c_{X}$ is a continuous function on $T(X)$. 
Proof. Let $\gamma(t)$ be a $C^{1}$ curve in $X$, defined in a neighborhood of 0 , with $\gamma(0)=x$ and $\gamma^{\prime}(0)=v$. For each $f$ in $H^{\infty}(X)$ we have

$$
\left(\phi_{*}(x) v\right)(f)=\lim _{t \rightarrow 0} \frac{\phi(\gamma(t))(f)-\phi(\gamma(0))(f)}{t}=\lim _{t \rightarrow 0} \frac{f(\gamma(t))-f(x)}{t}=f_{*}(x) v .
$$

Therefore $\phi_{*}(x) v$ is the linear functional that takes $f$ in $H^{\infty}(X)$ to $f_{*}(x) v$. By (4.4), the norm of that functional is $c_{X}(x, v)$.

The statements and proofs of the following lemma and its first corollary are implicit in Lewittes's paper [Lew66], particularly in the reasoning on its final page.

Lemma 4.3. If $a$ and $b$ are points of $\mathbb{D}$, then $|a-b| \leq 2 \tanh \frac{d_{\mathbb{D}}(a, b)}{2}$, with equality if and only if $b= \pm a$.

Proof. If $a=b$ there is nothing to prove. If $a \neq b$ choose a positive number $r<1$ and a conformal map $g$ of $\mathbb{D}$ onto itself so that $g(r)=a$ and $g(-r)=b$. Then $d_{\mathbb{D}}(a, b)=d_{\mathbb{D}}(r,-r)=2 d_{\mathbb{D}}(r, 0)$, so

$$
2 \tanh \frac{d_{\mathbb{D}}(a, b)}{2}=2 \tanh d_{\mathbb{D}}(r, 0)=2 r .
$$

It therefore suffices to prove that $|g(r)-g(-r)| \leq 2 r$ with equality if and only if $g(0)=0$. That is easily done by writing $g$ in the form

$$
g(z)=e^{i \theta}(z-\alpha) /(1-\bar{\alpha} z)
$$

with $|\alpha|<1$.

Corollary 4.4. $\|\phi(x)-\phi(y)\|=2 \tanh \frac{C_{X}(x, y)}{2}$ for all $x$ and $y$ in $X$,

Proof. Let $x$ and $y$ be given. For any $f$ in $H^{\infty}(X)$ with norm less than one, let $g_{f}$ be a conformal map of $\mathbb{D}$ onto itself that satisfies $g_{f}(f(x))=-g_{f}(f(y))$. Lemma 4.3 and the definition of $\phi$ give

$$
|(\phi(x)-\phi(y))(f)| \leq 2 \tanh \frac{d_{\mathbb{D}}(f(x), f(y))}{2}=\left|(\phi(x)-\phi(y))\left(g_{f} \circ f\right)\right| .
$$

The corollary follows by taking suprema over $f$.

We have already established the convention that if a curve $\gamma$ in $X$ is differentiable at some point $t$ in its parameter interval, then $\gamma^{\prime}(t)$ is the tangent vector $\gamma_{*}(t) 1$ to $X$ at $\gamma(t)$. It will be convenient from now on to use the same convention when $t$ is a complex variable and $\gamma$ is a holomorphic map from a neighborhood of $t$ to $X$.

Corollary 4.5. If $t \mapsto \gamma(t)$ is either a $C^{1}$ map of an open interval $(-\epsilon, \epsilon)$ into $X$ or a holomorphic map of an open disk $\{t \in \mathbb{C}:|t|<\epsilon\}$ into $X$, then (4.5) holds.

Proof. Under either hypothesis on $\gamma$, Corollary 4.4 and Lemma 4.1 imply that

$$
\lim _{t \rightarrow 0} \frac{C_{X}(\gamma(0), \gamma(t))}{|t|}=\lim _{t \rightarrow 0} \frac{2}{|t|} \tanh ^{-1} \frac{\|\phi(\gamma(0))-\phi(\gamma(t))\|}{2}=\left\|(\phi \circ \gamma)^{\prime}(0)\right\|,
$$

which equals $c_{X}\left(\gamma(0), \gamma^{\prime}(0)\right)$ by Corollary 4.2 . 
4.3. The Carathéodory-Reiffen pseudometric. In general $C_{X}(x, y)$ cannot be defined as the infimum of lengths of curves joining $x$ to $y$. In fact there are bounded domains of holomorphy $X$ in $\mathbb{C}^{2}$ such that not all open $C_{X}$ balls are connected (see [JP93]). For that reason the pseudometric $\widetilde{C}_{X}$ generated by the infinitesimal Carathéodory metric $c_{X}$ has independent interest.

Since it was first systematically studied (in the finite dimensional case) by Reiffen in [Ref65], $\widetilde{C}_{X}$ is called the Carathéodory-Reiffen pseudometric on $X$. It is defined in the obvious way. The Carathéodory length of a piecewise $C^{1}$ curve $\gamma:[a, b] \rightarrow X$ in $X$ is

$$
\widetilde{L}_{X}(\gamma)=\int_{a}^{b} c_{X}\left(\gamma(t), \gamma^{\prime}(t)\right) d t
$$

and the distance $\widetilde{C}_{X}(x, y)$ is the infimum of the lengths of all piecewise $C^{1}$ curves joining $x$ to $y$. Observe that the integrand in (4.6) is piecewise continuous.

Since $c_{\mathbb{D}}$ is the infinitesimal Poincaré metric on $\mathbb{D}$, its integrated form $\widetilde{C}_{\mathbb{D}}$ is the Poincaré metric. In addition, it follows readily from the definitions (4.4) and (4.6) that $\widetilde{L}_{Y}(f \circ \gamma) \leq \widetilde{L}_{X}(\gamma)$ for every piecewise $C^{1}$ curve in $X$ and $f \in \mathcal{O}(X, Y)$. Therefore the functor assigning $\widetilde{C}_{X}$ to each complex Banach manifold $X$ is a Schwarz-Pick system. In particular, if $x$ and $y$ are points in $X$, then

$$
d_{\mathbb{D}}(f(x), f(y)) \leq \widetilde{C}_{X}(x, y) \quad \text { for all } f \text { in } \mathcal{O}(X, \mathbb{D}) .
$$

Definition (4.3) therefore implies that

$$
C_{X}(x, y) \leq \widetilde{C}_{X}(x, y) \quad \text { for all } x \text { and } y \text { in } X .
$$

The relationship between $\widetilde{C}_{X}$ and $C_{X}$ is explored more fully in [Din89], [Har79], and [JP93].

\section{Distortion THEOREMS FOR COMPLEX NON-GEODESICS}

5.1. Complex geodesics. Since $X \mapsto \widetilde{C}_{X}$ is a Schwarz-Pick system, $\widetilde{C}_{X}$ is a Schwarz-Pick pseudometric on $X$ for every complex Banach manifold $X$. Therefore $\widetilde{C}_{X} \leq K_{X}$ for every $X$. Combining that inequality with (4.7) we obtain

$$
C_{X}(f(z), f(w)) \leq \widetilde{C}_{X}(f(z), f(w)) \leq K_{X}(f(z), f(w)) \leq d_{\mathbb{D}}(z, w)
$$

whenever $X$ is a complex Banach manifold, $f \in \mathcal{O}(\mathbb{D}, X)$, and $z$ and $w$ are points of $\mathbb{D}$.

Following Vesentini [Ves81], we call $f$ in $\mathcal{O}(\mathbb{D}, X)$ a complex geodesic (more precisely a complex $C_{X}$-geodesic) if there is a pair of distinct points $z$ and $w$ in $\mathbb{D}$ with

$$
C_{X}(f(z), f(w))=d_{\mathbb{D}}(z, w),
$$

so that none of the inequalities in (5.1) is strict. By a theorem of Vesentini (see Proposition 3.3 in [Ves81]), if $f$ is a complex geodesic then in fact (5.2) holds for all $z$ and $w$ in $\mathbb{D}$ (see also [Din89], [JP93], and [Ves82]).

In other words, if the inequality $C_{X}(f(z), f(w))<d_{\mathbb{D}}(z, w)$ holds for some pair of points then it must hold for all pairs of distinct points in $\mathbb{D}$. Our results in this section study quantitatively how the distortion of distance at one pair of points influences the degree of distortion at another. 
5.2. The Beardon-Minda quotient. The prototypes for our theorems can be found in the paper [BMi], where Beardon and Minda make an elegant systematic study of the situation when the target manifold $X=\mathbb{D}$. Their results indicate that the distortion of distance is appropriately measured by the quotient

$$
Q_{X}(f, a, b)=\frac{\tanh C_{X}(f(a), f(b))}{\tanh d_{\mathbb{D}}(a, b)},
$$

where $f \in \mathcal{O}(\mathbb{D}, X)$ and $a$ and $b$ are distinct points of $\mathbb{D}$. We call $Q_{X}(f, a, b)$ the Beardon-Minda quotient because for $f$ in $\mathcal{O}(\mathbb{D}, \mathbb{D})$ the number $Q_{\mathbb{D}}(f, a, b)$ is the absolute value of the "hyperbolic difference quotient" $f^{*}(a, b)$ on which Beardon and Minda base their study (see section 2 of $[\mathrm{BMi}]$ ) .

It is obvious that $0 \leq Q_{X}(f, a, b) \leq 1$, and $Q_{X}(f, a, b)=1$ if and only if $f$ is a complex geodesic. The size of $Q_{X}$ provides a quantitative measure of how close $f$ is to being a complex geodesic.

Our next result is inspired by and follows readily from Theorem 3.1 of [BMi]. Its proof provides a new proof (without use of normal family arguments) of Vesentini's theorem.

Theorem 5.1. If $X$ is a complex Banach manifold, $f \in \mathcal{O}(\mathbb{D}, X)$, and equation (5.2) fails for some pair of points in $\mathbb{D}$, then $Q_{X}(f, a, b)<1$ whenever the points a and $b$ in $\mathbb{D}$ are distinct. Moreover, if $a, b$, and $c$ are points of $\mathbb{D}$ and neither $b$ nor $c$ equals $a$, then

$$
d_{\mathbb{D}}\left(Q_{X}(f, a, b), Q_{X}(f, a, c)\right) \leq d_{\mathbb{D}}(b, c) .
$$

5.3. Proof of Theorem 5.1. Assume first that $X=\mathbb{D}$. In this case $C_{X}=d_{\mathbb{D}}$ and Theorem 5.1 reduces to a weak version of Theorem 3.1 in [BMi]. For the reader's convenience we include its proof, imitating the proof in [BMi].

By pre- and post-composing $f$ with appropriate Poincaré isometries, we may assume that $a=f(a)=0$. Then $Q_{\mathbb{D}}(f, 0, z)=|f(z)| /|z|$ for all nonzero $z$ in $\mathbb{D}$, so the conclusion of Theorem 5.1 reduces to the inequalities $|f(b)|<|b|$ for all nonzero $b$ in $\mathbb{D}$ and

$$
d_{\mathbb{D}}\left(\frac{|f(b)|}{|b|}, \frac{|f(c)|}{|c|}\right) \leq d_{\mathbb{D}}(b, c) \text { for all nonzero } b \text { and } c \text { in } \mathbb{D} .
$$

Since $f(0)=0$ and $f$ is not a rotation, the first inequality follows immediately from Schwarz's lemma. To obtain the second, define $g$ in $\mathcal{O}(\mathbb{D}, \mathbb{D})$ by the formula

$$
g(0)=f^{\prime}(0) \quad \text { and } \quad g(z)=f(z) / z \text { if } 0<|z|<1,
$$

apply the Schwarz-Pick lemma to $g$, and observe that $d_{\mathbb{D}}\left(|\zeta|,\left|\zeta^{\prime}\right|\right) \leq d_{\mathbb{D}}\left(\zeta, \zeta^{\prime}\right)$ for all $\zeta$ and $\zeta^{\prime}$ in $\mathbb{D}$.

Now we consider the general case. Given $f$ in $\mathcal{O}(\mathbb{D}, X)$ we choose any pair of distinct points $a$ and $b$ in $\mathbb{D}$. Suppose $Q_{X}(f, a, b)<1$.

Given any $g$ in $\mathcal{O}(X, \mathbb{D})$, set $h=g \circ f$. Then $Q_{\mathbb{D}}(h, a, b)<1$, so (by what we already proved) for all $c$ in $\mathbb{D} \backslash\{a\}$ we have $Q_{\mathbb{D}}(h, a, c)<1$ and

$$
d_{\mathbb{D}}\left(Q_{\mathbb{D}}(h, a, b), Q_{\mathbb{D}}(h, a, c)\right) \leq d_{\mathbb{D}}(b, c) .
$$

Set $k=\tanh d_{\mathbb{D}}(b, c), r=Q_{\mathbb{D}}(h, a, b)$, and $\widehat{r}=Q_{X}(f, a, b)$. By hypothesis these three numbers all lie in the half-open interval $[0,1)$. In terms of $k$ and $r,(5.5)$ becomes the double inequality 


$$
\frac{r-k}{1-k r} \leq Q_{\mathbb{D}}(h, a, c) \leq \frac{r+k}{1+k r} .
$$

Recall that $h=g \circ f$ for some $g$ in $\mathcal{O}(X, \mathbb{D})$. Taking suprema in (5.6) over all such $g$, we obtain

$$
\frac{\widehat{r}-k}{1-k \widehat{r}} \leq Q_{X}(f, a, c) \leq \frac{\widehat{r}+k}{1+k \widehat{r}} .
$$

Since $0 \leq \widehat{r}<1,(5.7)$ implies the inequalities $Q_{X}(f, a, c)<1$ and (5.4).

We have proved that if $Q_{X}(f, a, b)<1$ for some $a$ and $b$ in $\mathbb{D}$ then for all $c$ in $\mathbb{D} \backslash\{a\}$ we have both $Q_{X}(f, a, c)<1$ and the inequality (5.4). Theorem 5.1 follows readily.

5.4. Two corollaries of Theorem 5.1. In [BMi] Beardon and Minda derive many consequences of their Theorem 3.1. Some of them require only the inequality (5.5) and can therefore be generalized to our situation. We shall concentrate on results that involve the distortion of the infinitesimal Carathéodory pseudometric at points of $\mathbb{D}$. We measure that distortion by the Carathéodory norm of the derivative of the map $f$ in $\mathcal{O}(\mathbb{D}, X)$, which is defined as follows.

Definition 5.2. Let $X$ be a complex Banach manifold and let $f^{\prime}(a)=f_{*}(a) 1$ be the derivative of the map $f$ in $\mathcal{O}(\mathbb{D}, X)$ at the point $a$ in $\mathbb{D}$. The Carathéodory norm of $f^{\prime}(a)$ is the ratio of the Carathéodory length of the tangent vector 1 to $\mathbb{D}$ at $a$ and the Carathéodory length of its image in the tangent space to $X$ at $f(a)$.

Since it equals the limit of the Beardon-Minda quotient $Q_{X}(f, a, c)$ as $c$ approaches $a$ (see (5.10)), we shall denote the Carathéodory norm of $f^{\prime}(a)$ by the symbol $q_{X}(f, a)$. Explicitly,

$$
q_{X}(f, a)=\frac{c_{X}\left(f(a), f^{\prime}(a)\right)}{c_{\mathbb{D}}(a, 1)}=\left(1-|a|^{2}\right) c_{X}\left(f(a), f^{\prime}(a)\right) .
$$

For $f$ in $\mathcal{O}(\mathbb{D}, \mathbb{D})$ the number $q_{\mathbb{D}}(f, a)$ is the absolute value of the "hyperbolic derivative" $f^{h}(a)$ of Beardon and Minda (see section 2 of [BMi]).

Our next result quantifies Vesentini's theorem (Proposition 3.2 in [Ves81]) that if $f$ in $\mathcal{O}(\mathbb{D}, X)$ is not a complex geodesic then it shortens the Carathéodory lengths of all nonzero tangent vectors. For $f$ in $\mathcal{O}(\mathbb{D}, \mathbb{D})$ it is a weak special case of Theorem 3.1 in Beardon-Minda [BMi].

Corollary 5.3. If $X$ is a complex Banach manifold and $f$ in $\mathcal{O}(\mathbb{D}, X)$ is not a complex geodesic, then $q_{X}(f, a)<1$ for all $a$ in $\mathbb{D}$ and

$$
d_{\mathbb{D}}\left(Q_{X}(f, a, b), q_{X}(f, a)\right) \leq d_{\mathbb{D}}(a, b)
$$

whenever $a$ and $b$ are distinct points of $\mathbb{D}$.

Proof. Let $a$ and $b$ be distinct points of $\mathbb{D}$. As $Q_{X}(f, a, b)<1$ by hypothesis, (5.4) holds for any $c$ in $\mathbb{D} \backslash\{a\}$. We shall obtain (5.9) as the limiting case of (5.4) when $c \rightarrow a$ while $a$ and $b$ are held fixed.

As our first step, we apply Corollary 4.5 to the holomorphic maps $z \mapsto a+z$ and $z \mapsto f(a+z)$ from $\{z \in \mathbb{C}:|z|<1-|a|\}$ to $\mathbb{D}$ and $X$, obtaining the equations

$$
\lim _{z \rightarrow a} \frac{C_{X}(f(a), f(z))}{|z-a|}=c_{X}\left(f(a), f^{\prime}(a)\right)
$$


and

$$
\lim _{z \rightarrow a} \frac{d_{\mathbb{D}}(a, z)}{|z-a|}=c_{\mathbb{D}}(a, 1)\left(=\frac{1}{1-|a|^{2}}\right) .
$$

Using these equations, (5.3), and Definition 5.2, we obtain

$$
\lim _{c \rightarrow a} Q_{X}(f, a, c)=\lim _{c \rightarrow a} \frac{C_{X}(f(a), f(c))}{d_{\mathbb{D}}(a, c)}=\frac{c_{X}\left(f(a), f^{\prime}(a)\right)}{c_{\mathbb{D}}(a, 1)}=q_{X}(f, a) .
$$

Since $d_{\mathbb{D}}\left(Q_{X}(f, a, b), Q_{X}(f, a, c)\right) \leq d_{\mathbb{D}}(b, c) \leq d_{\mathbb{D}}(a, b)+d_{\mathbb{D}}(c, a)$ for all $c$ in $\mathbb{D} \backslash\{a\}$, it follows readily from $(5.10)$ that $q_{X}(f, a)$ belongs to $\mathbb{D}$ and satisfies (5.9).

For $f$ in $\mathcal{O}(\mathbb{D}, \mathbb{D})$ our next corollary is a restatement of Corollary 3.7 of Beardon and Minda [BMi]. The general case has essentially the same proof.

Corollary 5.4. If $X$ is a complex Banach manifold and $f$ in $\mathcal{O}(\mathbb{D}, X)$ is not a complex geodesic, then

$$
d_{\mathbb{D}}\left(q_{X}(f, a), q_{X}(f, b)\right) \leq 2 d_{\mathbb{D}}(a, b) \quad \text { for all } a \text { and } b \text { in } \mathbb{D} .
$$

Proof. If $a=b$ there is nothing to prove. If $a \neq b$ then Corollary 5.3 implies that the number

$$
d_{\mathbb{D}}\left(Q_{X}(f, a, b), q_{X}(f, a)\right)+d_{\mathbb{D}}\left(Q_{X}(f, a, b), q_{X}(f, b)\right)
$$

is bounded by $2 d_{\mathbb{D}}(a, b)$, so (5.11) follows from the triangle inequality.

5.5. The $\widetilde{C}_{X}$ version of Corollary 5.3. We do not know whether Theorem 5.1 remains valid when $C_{X}$ is replaced by $\widetilde{C}_{X}$ in its conclusion, but Corollary 5.3 does, as we shall now prove. Our method of proof is to use the inequality (5.11) to bound the Carathéodory length of appropriate curves in $X$. It was suggested to us in a private communication from David Minda about the classical case when $X=\mathbb{D}$.

Theorem 5.5. If $X$ is a complex Banach manifold and $f$ in $\mathcal{O}(\mathbb{D}, X)$ is not a complex $C_{X}$-geodesic, then $\widetilde{C}_{X}(f(a), f(b))<d_{\mathbb{D}}(a, b)$ and

$$
d_{\mathbb{D}}\left(\frac{\tanh \widetilde{C}_{X}(f(a), f(b))}{\tanh d_{\mathbb{D}}(a, b)}, q_{X}(f, a)\right) \leq d_{\mathbb{D}}(a, b) .
$$

whenever the points $a$ and $b$ in $\mathbb{D}$ are distinct.

Proof. Let distinct points $a$ and $b$ in $\mathbb{D}$ be given. Setting $k=\tanh d_{\mathbb{D}}(a, b)$ and $r=q_{X}(f, a)$, we can write $(5.12)$ as a double inequality

$$
k \frac{r-k}{1-r k} \leq \tanh \widetilde{C}_{X}(f(a), f(b)) \leq k \frac{r+k}{1+r k} .
$$

By hypothesis, $0<k<1$ and (by Corollary 5.3) $0 \leq r<1$. Therefore the right side of (5.13) is less than $k$, so (5.13) implies both $\widetilde{C}_{X}(f(a), f(b))<d_{\mathbb{D}}(a, b)$ and (5.12). We shall prove (5.13).

In terms of $r$ and $k$, the known inequality (5.9) can be written in the form

$$
k \frac{r-k}{1-r k} \leq \tanh C_{X}(f(a), f(b)) \leq k \frac{r+k}{1+r k} .
$$

Since $C_{X}(f(a), f(b)) \leq \widetilde{C}_{X}(f(a), f(b))$, the left side of (5.14) implies the left side of (5.13) but the right side of (5.13) requires proof. 
For the proof we may pre-compose $f$ with a Poincaré isometry so that $a=0$ and $b>0$. Then $b=\tanh d_{\mathbb{D}}(0, b)=k$ and the curve $\gamma(t)=f(t), 0 \leq t \leq k$, joins $f(a)$ to $f(b)$. Therefore

$$
\widetilde{C}_{X}(f(a), f(b)) \leq \widetilde{L}_{X}(\gamma)=\int_{0}^{k} c_{X}\left(f(t), f^{\prime}(t)\right) d t=\int_{0}^{k} \frac{q_{X}(f, t)}{1-t^{2}} d t,
$$

and it suffices to prove the inequality

$$
\int_{0}^{k} \frac{q_{X}(f, t)}{1-t^{2}} d t \leq \tanh ^{-1}\left(k \frac{r+k}{1+r k}\right) .
$$

Following a suggestion of David Minda, we use the identity

$$
\tanh ^{-1}\left(k \frac{r+k}{1+r k}\right)=\int_{0}^{k} \frac{r\left(1+t^{2}\right)+2 t}{\left(1-t^{2}\right)\left(1+2 r t+t^{2}\right)} d t .
$$

Comparison of the integrands in (5.15) and (5.16) shows that it suffices to prove that

$$
q_{X}(f, t) \leq \frac{r\left(1+t^{2}\right)+2 t}{1+2 r t+t^{2}} \quad \text { if } 0<t<1 .
$$

If $X=\mathbb{D}$ and $f(0)=0,(5.17)$ is contained in the inequality (6.1) in Beardon and Minda [BMi]. For the general case, we imitate their proof, using our Corollary 5.4 as follows. If $0<t<1$, then

$$
2 d_{\mathbb{D}}(0, t)=d_{\mathbb{D}}(-t, t)=d_{\mathbb{D}}\left(0, \frac{2 t}{1+t^{2}}\right)
$$

and (by definition of $r$ )

$$
d_{\mathbb{D}}\left(q_{X}(f, 0), q_{X}(f, t)\right)=d_{\mathbb{D}}\left(r, q_{X}(f, t)\right)=d_{\mathbb{D}}\left(0, \frac{q_{X}(f, t)-r}{1-r q_{X}(f, t)}\right) .
$$

Therefore, by Corollary $5.4, d_{\mathbb{D}}\left(0, \frac{q_{X}(f, t)-r}{1-r q_{X}(f, t)}\right) \leq d_{\mathbb{D}}\left(0, \frac{2 t}{1+t^{2}}\right)$, so

$$
\left|\frac{q_{X}(f, t)-r}{1-r q_{X}(f, t)}\right| \leq \frac{2 t}{1+t^{2}} \quad \text { if } 0<t<1 .
$$

The required inequality (5.17) follows readily.

The qualitative part of Theorem 5.5 provides the following strengthening of Proposition 3.3 in [Ves81].

Corollary 5.6. If $X$ is a complex Banach manifold, $f \in \mathcal{O}(\mathbb{D}, X)$, and the equation $\widetilde{C}_{X}(f(a), f(b))=d_{\mathbb{D}}(a, b)$ holds for some pair of distinct points $a$ and $b$ in $\mathbb{D}$, then $f$ is a complex $C_{X}$-geodesic.

Remark 5.7. In [BMi] Beardon and Minda show that the inequalities (5.4), (5.9), (5.11), and (5.12) are already sharp when $X=\mathbb{D}$. For example, equality occurs in all four of them if $f(z)=z^{2}, a=0$, and $c$ in (5.4) is a positive multiple of $b$. The cases of equality are fully analysed in [BMi].

Remark 5.8. As Theorems 5.1 and 5.5 illustrate, $C_{X}$ and $\widetilde{C}_{X}$ are on an equal footing in the theory of complex geodesics. If $f$ in $\mathcal{O}(\mathbb{D}, X)$ preserves the distance between two distinct points in either pseudometric, then it preserves the distance between any two points in both pseudometrics. 
The Kobayashi pseudometric, however, stands apart. The methods we have used in this section do not apply to it, and simple examples show that the stated results cannot be extended to the Kobayashi pseudometric without additional hypotheses. For instance, holomorphic universal covering maps are known (see [Din89] or [Kob98]) to be local isometries in the Kobayashi pseudometric, so if $X$ is any hyperbolic Riemann surface other than $\mathbb{D}$ none of the theorems and corollaries in this section holds true when $C_{X}$ or $\widetilde{C}_{X}$ is replaced by $K_{X}$.

One might ask whether $K_{X}$ can be substituted for $C_{X}$ or $\widetilde{C}_{X}$ in these results if $X$ is required to be simply connected, but there are difficulties even for contractible domains of holomorphy in $\mathbb{C}^{n}, n>1$. For example, let

$$
X=\left\{(z, w) \in \mathbb{C}^{2}:|z|<1,|w|<1, \text { and }|z w|<a^{2}\right\},
$$

where $0<a<\frac{1}{2}$. Vigué [Vig85] remarks that the map $\zeta \mapsto(a \zeta, a \zeta)$ from $\mathbb{D}$ to $X$ preserves the Kobayashi lengths of the tangent vectors at $\zeta=0$ but decreases the Kobayashi distances from 0 . Thus the $K_{X}$ version of Corollary 5.3 fails for holomorphic maps of $\mathbb{D}$ into this domain $X$.

But Theorem 5.1 could still hold, for Venturini uses the same map in [Ven89] to show that the infinitesimal Kobayashi metric is not the derivative of $K_{X}$ at the origin in $X$. The limiting argument by which we obtained Corollary 5.3 from Theorem 5.1 therefore fails in this case, and the question whether some form of Theorem 5.1 holds for $K_{X}$ in this domain appears to be still open.

Remark 5.9. Samuel Krushkal's preprint [Kru01] contains the remarkable result that the Carathéodory and Teichmüller metrics on the universal Teichmüller space are equal. Using that result we can use the Teichmüller metric in the formulas for $Q_{X}$ and $q_{X}$ when $X$ is the universal Teichmüller space. The inequalities (5.4), (5.9), (5.11) then become remarkable strengthenings of the classical principle of Teichmüller contraction (see [Ear02]).

\section{REFERENCES}

[BMi] A. F. Beardon and D. Minda (in press) 'A multi-point Schwarz-Pick lemma', J. Anal. Math.

[Car26] C. Carathéodory (1926) 'Über das Schwarzsche Lemma bei analytischen Funktionen von zwei komplexen Veränderlichen', Math. Ann. 97, 76-98.

[Din89] S. Dineen (1989) 'The Schwarz Lemma', Oxford Mathematical Monographs, Clarendon Press, Oxford.

[Dou66] A. Douady (1966) 'Le probléme des modules pour les sous-espaces analytiques compacts d'un espace analytique donné', Ann. Inst. Fourier (Grenoble) 16, 1-95.

[Ear02] C. J. Earle (2002) 'Schwarz's lemma and Teichmüller contraction'. In Complex Manifolds and Hyperbolic Geometry, Contemp. Math. 311, 79-85, Amer. Math. Soc., Providence.

[EH70] C. J. Earle and R. S. Hamilton (1970) 'A fixed point theorem for holomorphic mappings'. In Global Analysis, Proc. Sympos. Pure Math. XVI, 61-65, Amer. Math. Soc., Providence.

[FV80] T. Franzoni and E. Vesentini (1980) 'Holomorphic Maps and Invariant Distances', NorthHolland Mathematical Studies 40, North-Holland Publishing, Amsterdam.

[Har79] L. A. Harris (1979) 'Schwarz-Pick systems of pseudometrics for domains in normed linear spaces'. In Advances in Holomorphy, North-Holland Mathematical Studies 34, 345-406, North-Holland Publishing, Amsterdam.

[JP93] M. Jarnicki and P. Pflug (1993) 'Invariant Distances and Metrics in Complex Analysis', de Gruyter Expositions in Mathematics 9, Walter de Gruyter, Berlin-New York.

[Kob67] S. Kobayashi (1967) 'Invariant distances on complex manifolds and holomorphic mappings', J. Math. Soc. Japan 19, 460-480.

[Kob98] S. Kobayashi (1998) 'Hyperbolic Complex Spaces', Grundlehren der Mathematischen Wissenschaften, Vol. 318, Springer-Verlag, Berlin. 
[Kru01] S. L. Krushkal (2001) 'Complex geometry of the universal Teichmüller space, Grunsky coefficients and plurisubharmonic functions', preprint.

[Lan62] S. Lang (1962) 'Introduction to Differentiable Manifolds', Wiley-Interscience, New York.

[Lew66] J. Lewittes (1966) 'A note on parts and hyperbolic geometry', Proc. Amer. Math. Soc. 17, 1087-1090.

[NO90] J. Noguchi and T. Ochiai (1990) 'Geometric Function Theory in Several Complex Variables', Translations of Mathematical Monographs, Vol. 80, Amer. Math. Soc., Providence.

[PSh89] E. A. Poletskii and B. V. Shabat (1989) 'Invariant metrics'. In Several Complex Variables III, Encyclopædia of Mathematical Sciences, Vol. 9, 63-111, Springer-Verlag, Berlin.

[Ref65] H.-J. Reiffen (1965) 'Die Carathéodory Distanz und ihre zugehörige Differentialmetric', Math. Ann. 161, 315-324.

[Roy71] H. L. Royden (1971) 'Remarks on the Kobayashi metric'. In Several Complex Variables II, Maryland 1970, Lecture Notes in Math. 185, 125-137, Springer-Verlag, Berlin.

[Roy74] H. L. Royden (1974) 'The extension of regular holomorphic maps', Proc. Amer. Math. Soc. 43, 306-310.

[Roy88] H. L. Royden (1988) 'Hyperbolicity in complex analysis', Ann. Acad. Sci. Fenn. 13, $387-400$.

[Ven89] S. Venturini (1989) 'Pseudodistances and pseudometrics on real and complex manifolds', Ann. Mat. Pura Appl. 154, 385-402.

[Ves81] E. Vesentini (1981) 'Complex geodesics', Compositio Math. 44, 375-394.

[Ves82] E. Vesentini (1982) 'Complex geodesics and holomorphic mappings', Sympos. Math. 26, $211-230$.

[Vig85] J.-P. Vigué (1985/86) 'Sur la caractérisation des automorphismes analytiques d'un domaine borné', Portugal. Math. 43, 439-453.

(Earle and Hubbard) Department of Mathematics, Cornell University, Ithaca, Ny $14853-7901$

(Harris) Department of Mathematics, University of Kentucky, Lexington, KY 40506 0027

(Mitra) Department of Mathematics, University of Connecticut, Storrs, CT 062693009

E-mail address, Earle: cliff@math.cornell.edu

E-mail address, Harris: larry@ms.uky.edu

E-mail address, Hubbard: hubbard@math.cornell.edu

E-mail address, Mitra: mitra@math.uconn.edu 